und nicht zuletzt all denjenigen, die ausländische Freiwillige, die bei uns einen Freiwilligendienst leisten, aufnehmen. Diese ideale Ausgangslage weiter auszubauen, die Chancen und Potentiale der Freiwilligendienste zu erkennen und mit allen gesellschaftlichen Akteuren zu nutzen, wird Aufgabe der nächsten Jahre sein.

Ich erhoffe mir von Voluntaris ein kreatives und unabhängiges Forum des Austausches über die vielen Herausforderungen in den verschiedenen Formaten. Ich wünsche mir eine Plattform zur fachlichen und sachlichen Auseinandersetzung mit Akteuren aus Wissenschaft, Praxis und Politik, aber auch den Blick auf die Grenzen der Freiwilligendienste und über sie hinaus. Ich erwarte einen spannenden Erfahrungsaustausch mit engagierten Vertreterinnen und Vertretern aus Wohlfahrtsverbänden, Kirchen und Zivilgesellschaft, mit Praktikerinnen und Praktikern aus Trägern und Einsatzstellen und, last but not least, mit Freiwilligen aller Altersgruppen und möglicher Dienstformen.

\title{
Bundesministerium für wirtschaftliche Zusammenarbeit und Entwicklung (BMZ)
}

\author{
Hans-Jürgen Beerfeltz
}

Staatssekretär des Bundesministeriums für wirtschaftliche Zusammenarbeit und Entwicklung |hans-juergen.beerfeltz@bmz.bund.de|www.bmz.de

Für Entwicklungszusammenarbeit ist das tatkräftige Engagement von Freiwilligen unverzichtbar. Denn: Der Staat kann die

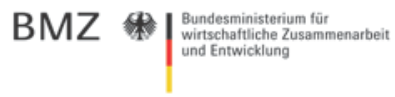
vielfältigen Herausforderungen nicht alleine bewältigen. Staat, Wirtschaft und die Zivilgesellschaft erfüllen je eigenständige Funktionen, die sich nicht wechselseitig substituieren lassen. Freiwillige bringen besondere Kreativität, Leidenschaft und Ausdauer mit. Zudem sind sie wichtige Multiplikatoren für die Anliegen der Entwicklungszusammenarbeit in der deutschen Gesellschaft: Sie erzählen ihren Familien, Freunden, Kolleginnen und Kollegen von ihren Erfahrungen. So wird Entwicklungszusammenarbeit auch erlebbar für jene, die noch nicht engagiert sind. 
Eines unserer jüngsten Erfolgsmodelle für Freiwilligenarbeit ist weltwärts. Seit 2008 haben über 16.500 junge Freiwillige in mehr als 70 Ländern ihren Einsatz begonnen. Aktuell unterstützen die rund 3200 Freiwilligen Initiativen in den Kooperationsländern in ihrer täglichen Arbeit und nehmen dabei einiges für ihr späteres Leben mit. Sie lernen etwas über sich selbst, über die Menschen und Lebensbedingungen in den Kooperationsländern sowie über den Wert ehrenamtlichen Engagements. Weltwärts aktiviert junge Menschen zwischen 18 und 28 Jahren, die uns immer wieder begeistert von ihrer Arbeit und ihren Erfahrungen vor Ort berichten. Sie hinterfragen dabei gleichzeitig kritisch ihre eigene entwicklungspolitische Rolle. Neben weltwärts fördert das BMZ weitere Programme, die entwicklungspolitisches Engagement stärken. Das ASA-Programm ist eine Lernwerkstatt für entwicklungspolitisch interessierte junge Menschen und der Senior Expert Service bietet Menschen im Ruhestand die Möglichkeit, ihre beruflichen Kenntnisse an andere im Ausland weiterzugeben. Diese Programme unterscheiden sich in ihrer Ausrichtung, Zielgruppe und Zielsetzung. Gemein ist ihnen jedoch, dass sie freiwilliges Engagement der deutschen Zivilgesellschaft in der Entwicklungspolitik fördern und unterstützen. Mit Engagement Global wurde darüber hinaus ein zentraler Dienstleister als „One-Stop-Shop“ für die Zivilgesellschaft geschaffen, der Menschen zu mehr Engagement ermutigt und hilft, administrative Hürden zu überwinden oder eine Förderung zu beantragen. Die BMZ-geförderten Freiwilligendienste werden permanent weiterentwickelt. So entfalten sie für die Teilnehmenden und unsere Partner die größte Wirkung. Es sind lernende Programme, die im Austausch mit den Teilnehmenden und den zivilgesellschaftlichen Stakeholdern laufend justiert werden.

Mit der Zeitschrift Voluntaris wird eine Plattform geschaffen, die den Austausch zwischen Wissenschaft, Politik und Praxis rund um Freiwilligendienste ermöglicht. Eine derartige Plattform gibt es in Deutschland bis dato nicht, ihre Gründung begrüße ich daher ausdrücklich.

$\mathrm{Zu}$ einer kritikfähigen Praxis und einer zielorientierten Wissenschaft gehört auch eine kritikfähige und innovative Politik, die das Umfeld und die Rahmenbedingungen für entwicklungspolitisches Engagement bereitstellt. Unsere Programme sind sehr erfolgreich, stehen aber auch vor Herausforderungen, die wir angehen müssen. Erkenntnisse, wie wir sie durch die Evaluierung unserer Freiwilligendienstprogramme gewonnen haben, können in 
Voluntaris einer weitergehenden Betrachtung unterzogen werden. Als Fördergeber möchte das BMZ ein Umfeld schaffen, in dem auch offen über weniger erfolgreiche Erfahrungen und Entwicklungen geredet wird. Nur das ermöglicht uns, gemeinsam voneinander zu lernen. Ich wünsche mir, dass Voluntaris die Analyse und den Austausch über die Entwicklungen rund um entwicklungspolitische Freiwilligendienste begleitet und vorantreibt.

Zum Start wünsche ich der Zeitschrift viel Erfolg und eine breite Leserinnen- und Leserschaft!

\title{
Bundesnetzwerk Bürgerschaftliches Engagement (BBE)
}

\author{
PD Dr. Ansgar Klein
}

Geschäftsführer des Bundesnetzwerks Bürgerschaftliches Engagement ansgar.klein@b-b-e.de|www.b-b-e.de

Im Mai 2013 startet die neue Fachzeitschrift Voluntaris. Sie kommt zur rechten Zeit! Niemals zuvor haben die Freiwilligendienste in Deutschland so große Bedeutung gehabt. Niemals zuvor gab es so viele Teilnehmer und

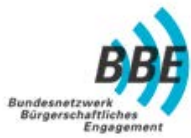
ein so großes Angebotsfeld an Freiwilligendiensten. Und niemals zuvor bestand - nach Gründung des neuen Bundesfreiwilligendienstes und der Umwidmung des ehemaligen Bundesamtes für Zivildienst zum neuen Bundesamt für Familie und zivilgesellschaftliche Aufgaben (,BAFzA“) - so viel Gesprächs- und Diskursbedarf.

Voluntaris hat vor diesem Hintergrund die große Chance und Aufgabe, die bestehenden Fachdiskurse rund um die Freiwilligendienste ebenso aufzunehmen wie die praktischen Erfahrungen derer, die einen der vielen Dienste absolviert haben, zu berichten und auszuwerten.

Folgende Themen werden in einer neuen Fachzeitschrift zu Freiwilligendiensten insbesondere erwartet: 